\title{
Convective Nonlinearity in Non-Newtonian Fluids
}

\author{
Hubert Temmen, ${ }^{1}$ Harald Pleiner, ${ }^{2}$ Mario Liu ${ }^{3}$ and Helmut R. Brand ${ }^{4}$
}

1. EMA, Universität der Bundeswehr Hamburg, Holstenhofweg 85, 22043 Hamburg

2. Max-Planck-Institut für Polymerforschung, Postfach 3148, 55021 Mainz

3. Institut für Theoretische Physik, Universität Hannover, 30167 Hannover

4. Theoretische Physik III, Universität Bayreuth, 95440 Bayreuth, Germany

(PRL 84, 3223, (2000))

In the limit of infinite yield time for stresses, the hydrodynamic equations for viscoelastic, Non-Newtonian liquids such as polymer melts must reduce to that for solids. This piece of information suffices to uniquely determine the nonlinear convective derivative, an ongoing point of contention in the rheology literature.

05.70.Ln, 46.05.+b,83.10.Nn

Hydrodynamics, the macroscopic description of condensed systems in the low frequency, long wavelength limit [1,2], is a well-established, systematic approach in condensed matter physics. This method has been applied over the past decades to many systems, including simple fluids [3], various liquid crystals [1, 4], and the superfluid phases of ${ }^{3} \mathrm{He}$ and ${ }^{4} \mathrm{He}[5]$.

All viscoelastic non-Newtonian fluids behave as Newtonian ones at low frequencies, and as solids at higher frequencies. A consistent hydrodynamic description needs to reflect this fact and must therefore contain, as special cases, both the hydrodynamic theory for isotropic liquids and solids. The liquid limit is well heeded in the polymer literature and universally correctly implemented [6.7]. The solid limit is problematic, as we shall see, and compatibility especially in the nonlinear regime of large displacements and rotations has so far proven elusive. The reason behind it is probably the lack of a consistent hydrodynamic theory for solids.

The last sentence may come as a surprise, but the point we are making here is: Although both the nonlinear elasticity theory [6] and the linearized hydrodynamics for crystals [1, 8, 9] are well known and established, a consistent hydrodynamic theory that includes both nonlinear and irreversible terms is not - in spite of some insightful papers [10]. One of the obstacles is that such a theory necessarily employs a strain tensor different from the one customarily used [8]. The usual strain tensor is of the Lagrange type, derived from equations of motion for mass points, while a framework to set up hydrodynamic equations including dissipative terms only exists in the Eulerian description - which considers evolution of field variables at spatial points. Consistency forbids a mixing of both descriptions and requires an Eulerian strain tensor [11]. (We note that the linear hydrodynamic theory may mix both descriptions, as the smallness of the displacements ensures that the discrepancy is negligible.)

The presentation of the nonlinear hydrodynamic theory for solids is what we shall do first. Then these equations are generalized for non-Newtonian fluids by adding relaxation-type terms to account for a finite yield time of the stresses, such that in the high frequency limit the theory is unchanged, but in the low frequency limit only the terms of the isotropic liquid hydrodynamics remain. So, by ensuring the valid liquid and solid limits, this approach leads to the correct hydrodynamic theory for any liquids displaying viscoelasticity. It is of great importance for rheology, as many competing theories exist, which differ especially in their respective nonlinear convective derivatives. All are employed in the literature, with scant hope for consensus.

Comparing our result with the literature, we find that none of the convective nonlinearities suggested in the rheology literature [6.7] contains the correct solid-limit, putting justified doubts on their validity. (We do obtain, for the Eulerian strain and in the limit in which it is small, the so called "upper convected derivative".)

Note that the insistence on the solid limit also determines the choice of variables, being that of a solid: the strain and the conserved quantities. As the solid is the more complicated of the two limits, there is no reason to, in addition, take the stress as an independent variable, as most theories in the rheology literature do, see for instance Chap. 7-9 in the first of [7]. Aside from unnecessarily making a derived quantity independent, this approach also commits a cardinal hydrodynamic sin, because the stress as a hydrodynamic flux contains reactive and dissipative parts, and does not possess a well-defined time reversal parity - without which we have no way of applying the Onsager relations.

We now introduce the nonlinear hydrodynamic theory of solids. A proper description relies on two coordinates: the actual spatial coordinate $r_{i}$, specifying a point in an elastic body, and the coordinate $a_{i}$ this point possesses in the absence of any stresses. More carefully, starting from a stress-free elastic body, we consider a point with the initial coordinate $a_{i}$. As the body is translated, rotated, compressed and sheared, this point is displaced to $r_{i}-$ especially in soft matter generally rather remote from $a_{i}$. Since all points of the body have a unique pair of $a_{i}$ and $r_{i}$, the function $r_{i}\left(a_{m}\right)$ is unique and invertible, the result of which is denoted as $a_{i}\left(r_{m}\right)$. For briefness, we shall refer to all $r_{i}$ as the real space, and to all $a_{i}$ as the 
initial space.

As discussed in most books on elasticity theory, see eg [8], the elastic energy depends on the change in the distance between any two neighbouring points, from $\mathrm{d} a_{i}^{2}$ to $\mathrm{d} r_{i}^{2}$. Defining the displacement vector as $u_{i}\left(a_{m}\right)=$ $r_{i}\left(a_{m}\right)-a_{i}$, and the strain tensor as $U_{i k}^{L}=\frac{1}{2}\left[\partial u_{i} / \partial a_{k}+\right.$ $\left.\partial u_{k} / \partial a_{i}+\left(\partial u_{j} / \partial a_{i}\right) \cdot\left(\partial u_{j} / \partial a_{k}\right)\right]$, we have $\mathrm{d} r_{i}^{2}\left(a_{m}\right)-$ $\mathrm{d} a_{i}^{2}=2 U_{i k}^{L} \mathrm{~d} a_{i} \mathrm{~d} a_{k}$, and know to take the energy density as a function of $U_{i k}^{L}$, to lowest order simply as $\epsilon=\frac{1}{2} K_{i k j m} U_{i k}^{L} U_{j m}^{L}$. The important point here is that both the strain tensor and the energy density are functions of the initial coordinate $a_{m}$, a notation that we shall refer to as Lagrangian - hence the superscript in $U_{i k}^{L}$.

Contrast this with the energy density of an isotropic liquid in its rest frame, a function of the mass and entropy density, $\epsilon(\rho, s)-$ or equivalently, $\mathrm{d} \epsilon=T \mathrm{~d} s+\mu \mathrm{d} \rho$. All variables, including the conjugate ones, temperature $T$ and chemical potential $\mu$, are here functions of the real coordinate $r_{m}$. As a result, the spatial dependence of (say) the temperature is quite independent of the liquid's compressional state. This is the Euler notation, and its basic advantage is that physics, which we insist must be local, is also expressed in local terms, accounted for by quantities at the real coordinates $r_{m}$. Consider for instance the diffusive heat current, which is given by the local gradient of the temperature, $\sim \partial T\left(r_{m}\right) / \partial r_{k}$, only in the Eulerian description.

Returning to solids, we have two choices: First, take all variables including especially the temperature and chemical potential as functions of $a_{m}$, and employ them with the strain tensor $U_{i k}^{L}$. This would be consistent, but highly inconvenient. For instance, the heat current $\sim \partial T\left(r_{m}\right) / \partial r_{i}$ at the real space point $r_{m}$ now presumes the knowledge (not usually available) of the global transformation, $r_{m} \leftrightarrow a_{m}$, as $\partial T\left(r_{m}\right) / \partial r_{i}=$ $\left[\partial T\left(a_{m}\right) / \partial a_{k}\right]\left(\partial a_{k} / \partial r_{i}\right)$. Similarly, with $\boldsymbol{g}$ the momentum density, the angular momentum density is $\mathbf{r}\left(a_{m}\right) \times$ $\boldsymbol{g}\left(a_{m}\right)$ rather than $\boldsymbol{a} \times \boldsymbol{g}\left(a_{m}\right)$. (If the system is only weakly deformed, with $u_{i}=r_{i}-a_{i}$ small, the above differences between $r_{i}$ and $a_{i}$ may be neglected to linear order.) Finally, more specific to the issue at hand, our equations need to contain both the elasticity theory and the liquid hydrodynamics. The latter, however, is usually and concisely given in the Euler notation.

The second, and the only actually viable, choice is to take all variables including the strain tensor in the local, Eulerian notation, as functions of $r_{m}$. We shall therefore employ the Eulerian strain tensor [11]12], introduced via $\mathrm{d} r_{i}^{2}-\mathrm{d} a_{i}^{2}\left(r_{m}\right)=2 U_{i k}\left(r_{m}\right) \mathrm{d} r_{i} \mathrm{~d} r_{k}$, where $U_{i k}\left(r_{m}\right)=\frac{1}{2}\left[\partial u_{i} / \partial r_{k}+\partial u_{k} / \partial r_{i}-\left(\partial u_{j} / \partial r_{i}\right)\left(\partial u_{j} / \partial r_{k}\right)\right]$ and $u_{i}\left(r_{m}\right) \stackrel{1}{=} r_{i}-a_{i}\left(r_{m}\right)$.

In this context, there is a second, somewhat subtle point: We need to eliminate the displacement field $u_{i}$, and deal exclusively with the initial coordinate $a_{i}(r)$ and the strain $U_{i k}$ in the elasticity theory. This is possible because starting again from $\mathrm{d} r_{i}^{2}-\mathrm{d} a_{i}^{2}\left(r_{m}\right)=$ $2 U_{i k}\left(r_{m}\right) \mathrm{d} r_{i} \mathrm{~d} r_{k}$, we find that the strain tensor may be written as

$$
U_{i k}=\frac{1}{2}\left[\delta_{i k}-\left(\partial a_{\alpha} / \partial r_{k}\right)\left(\partial a_{\alpha} / \partial r_{i}\right)\right],
$$

with no need whatever for a detour via $u_{i}$. This is necessary because the introduction of $u_{i}$ destroys a symmetry and represents an arbitrary (gauge) choice. As discussed, $a_{i}$ and $r_{i}$ are vectors of different spaces, so they transform as vectors under rotations in initial and real space, respectively. The introduction of the displacement fixes both spaces with respect to each other, and prohibits the rotation of either space alone. On the other hand, the elastic energy is independent of the orientation of the initial space, the fictitious unstressed body. Given any transformation $a_{m} \leftrightarrow r_{m}$, we should still be free to take a global but arbitrary rotation of all $a_{i}$, ie, rotate the initial space with respect to the real space. Therefore, $a_{\alpha}$ and $r_{i}$ are indeed vectors of two different spaces, and a quantity such as $\nabla_{k} a_{\alpha} \equiv \partial a_{\alpha} / \partial r_{i}$ is a vector both in real and initial space, (a bi-vector,) and not a second rank tensor. We use Latin and Greek indices to denote the components $(\mathrm{x}, \mathrm{y}, \mathrm{z})$ in real space, and $(1,2,3)$ in initial space, respectively. (Clearly, this renders the fact that the displacement $r_{i}-a_{\alpha}$ is an oxymoron rather obvious.)

We now proceed to understand that the bi-vector $\nabla_{i} a_{\alpha}$ not only contains the information about the strain, as shown in Eq (1), but also that about the local orientation. The polar decomposition theorem (cf W. Noll, p.65 ff, Vol.2 of [6]) states

$$
\nabla_{i} a_{\alpha}=R_{\alpha j} \Xi_{i j},
$$

where $R_{\alpha j}$ is the rotation matrix that rotates the local preferred directions in real space back to the global ones in initial space, while $\Xi_{i j}$ is a symmetric matrix that deviates from $\delta_{i j}$ only for finite strains. Consider first the unstrained case $\Xi_{i j}=\delta_{i j}$ : Because of $\mathrm{d} a_{\alpha}=\left(\nabla_{i} a_{\alpha}\right) \mathrm{d} r_{i}$ with $\mathrm{d} a_{\alpha}^{2}=\mathrm{d} r_{i}^{2}$, the gradient $\nabla_{i} a_{\alpha}$ is indeed a rotation matrix $R_{\alpha j}$, and must satisfy $R_{\alpha j} R_{\alpha k}=\delta_{j k}$, $R_{\alpha j} R_{\beta j}=\delta_{\alpha \beta}$. For finite strains, Eq. (1) implies $\delta_{i j}-2 U_{i j}=R_{\alpha k} \Xi_{i k} R_{\alpha l} \Xi_{l j}=\Xi_{i k} \Xi_{k j}$, the square root of which is

$$
\Xi_{i j}=\sqrt{\delta_{i j}-2 U_{i j}} \approx\left(\delta_{i j}-U_{i j}-\frac{1}{2} U_{i k} U_{k j} \cdots\right) .
$$

[This expansion is valid for small strains $U_{i j}$, but arbitrary rotations $R_{\alpha j}$. The square root of a matrix is defined by its power series. One can verify Eq (3) by calculating $\Xi_{i k} \Xi_{k j}$.]

In accounting for solid behaviour, we need to keep track of the local preferred directions, or $R_{\alpha i}$, which may vary considerably by accumulation over a long distance, even if the strain is small - think of a sheet of single crystal, slightly bent over a long stretch to form a tube of large radius. Let us consider as an example the harmonic approximation for the energy $E=\int \epsilon \mathrm{d} V$,

$$
\begin{array}{r}
\epsilon=\frac{1}{2} K_{i j k m} U_{i j} U_{k m}=\frac{1}{2} K_{\alpha \beta \gamma \delta} U_{\alpha \beta} U_{\gamma \delta} \\
U_{i j}=R_{\alpha i} R_{\beta j} U_{\alpha \beta}, \quad K_{i k j m}=R_{\alpha i} R_{\beta j} R_{\gamma k} R_{\delta m} K_{\alpha \beta \gamma \delta}
\end{array}
$$


where $U_{\alpha \beta}$ and $K_{\alpha \beta \gamma \delta}$ are the attendant quantities in the initial space. ( $\epsilon$ has the same form in both spaces because $R_{\alpha i}$ annihilates pairwise.) The elements of $K_{\alpha \beta \gamma \delta}$ are constant. A cubic crystal for instance has three independent elements, of which one is given as $K_{1111}=$ $K_{2222}=K_{3333}$, implying that the compressional energy is the same along the three initial space directions 1, 2, and 3. This is not the case for a hexagonal crystal, for which the compressional energy along 3 is different from 1 or 2 . The real space matrix $K_{i j k m}$ depends on $R_{\alpha i}$ and varies in space, as $\mathrm{Eq}(5)$ shows, because the symmetry axis 3 (of a hexagonal crystal) may in real space point in any direction, and vary spatially. So $\epsilon$ is a function of $U_{i j}$ and $R_{\alpha i}$. Writing $\mathrm{d} \epsilon=\Psi_{i j} \mathrm{~d} U_{i j}+\chi_{\alpha i} \mathrm{~d} R_{\alpha i}$, the conjugate variables $\Psi_{i j}=K_{i j k m} U_{k m}$ and $\chi_{\alpha i}=2 R_{\beta j} R_{\gamma k} R_{\delta m}$ $K_{\alpha \beta \gamma \delta} U_{i j} U_{k m}$ are given by differentiating Eqs (4) and (5). The energy $\epsilon$ depends on $U_{i j}$ and $R_{\alpha i}$ beyond the validity of Eq (4), so $\mathrm{d} \epsilon=\Psi_{i j} \mathrm{~d} U_{i j}+\chi_{\alpha i} \mathrm{~d} R_{\alpha i}$ is generally valid - though the explicit form of $\Psi_{i j}$ and $\chi_{\alpha i}$ will vary.

The term $\chi_{\alpha i} d R_{\alpha i}$ is rarely included in the energy for solids [8], which renders the resultant formulas valid only for small deformations or isotropic solids. For the latter systems there is no preferred direction to keep track of locally, so $K_{i j k m}$ will not depend on $R_{\alpha i}$, and we can set $\chi_{\alpha i}=0$. This is easiest seen in the harmonic approximation, Eq (4), where $K_{\alpha \beta \gamma \delta}=\left(K_{L}-\right.$ $\left.K_{T} / 3\right) \delta_{\alpha \beta} \delta_{\gamma \delta}+K_{T} / 2\left(\delta_{\alpha \gamma} \delta_{\beta \delta}+\delta_{\alpha \delta} \delta_{\beta \gamma}\right)$ due to isotropy. Inserting this into Eq (5), we again obtain $K_{i j k m}=$ $\left(K_{L}-K_{T} / 3\right) \delta_{i j} \delta_{k m}+K_{T} / 2\left(\delta_{i k} \delta_{j m}+\delta_{i m} \delta_{j k}\right)$, manifestly independent of $R_{\alpha i}$.

Returning to anisotropic systems, the 9 variables of $\nabla_{i} a_{\alpha}$ are equivalent to the 3 of $R_{\alpha i}$ and the 6 of $U_{i j}$ (or $\Xi_{i j}$ ), see Eqs. (2) and (3). So we can conveniently write $\Psi_{i j} \mathrm{~d} U_{i j}+\chi_{\alpha i} \mathrm{~d} R_{\alpha i}=\psi_{\alpha i} \mathrm{~d} \nabla_{i} a_{\alpha}$ where $\psi_{\alpha i}=\Psi_{k m}\left(\partial U_{k m} / \partial \nabla_{i} a_{\alpha}\right)+\chi_{\beta k}\left(\partial R_{\beta k} / \partial \nabla_{i} a_{\alpha}\right)$. Under a real space rotation of the angle $\mathrm{d} \theta_{i}$, scalars are invariant, $\mathrm{d} \epsilon=\psi_{\alpha i} \mathrm{~d} \nabla_{i} a_{\alpha}=0$, but vectors and tensors are not, $\mathrm{d} \nabla_{i} a_{\alpha}=\epsilon_{i j k} \nabla_{j} a_{\alpha} \mathrm{d} \theta_{k}$, so $\psi_{\alpha i} \epsilon_{i j k} \nabla_{j} a_{\alpha}=0$, or $\left(\psi_{\alpha j} \nabla_{i} a_{\alpha}\right)=(i \leftrightarrow j)$. Similarly, $\mathrm{d} R_{\alpha i}=\epsilon_{i j k} R_{\alpha j} \mathrm{~d} \theta_{k}$, $\mathrm{d} U_{i m}=\epsilon_{i j k} U_{j m} \mathrm{~d} \theta_{k}+\epsilon_{m j k} U_{i j} \mathrm{~d} \theta_{k}$, so $\left(\Psi_{i k} U_{j k}+\Psi_{k i} U_{k j}+\right.$ $\left.\chi_{\alpha i} R_{\alpha j}\right)=(i \leftrightarrow j)$. (These constraints on $\psi_{\alpha j}, \Psi_{k i}$, and $\chi_{\alpha i}$ can be used to show the symmetry of the stress tensor $\sigma_{i j}$ below.)

More generally, $\epsilon$ also depends on the mass, entropy and momentum density, $\rho, s$, and $g_{i}$. So the final thermodynamic expression for an elastic medium is

$$
\mathrm{d} \epsilon=T \mathrm{~d} s+\mu \mathrm{d} \rho+v_{i} \mathrm{~d} g_{i}+\psi_{\alpha i} \mathrm{~d} \nabla_{i} a_{\alpha} .
$$

Turning now to dynamics, the equation of motion for $a_{\alpha}$ is

$$
\frac{\mathrm{d}}{\mathrm{d} t} a_{\alpha} \equiv \dot{a}_{\alpha}+v_{k} \nabla_{k} a_{\alpha}=-Y_{\alpha} .
$$

In equilibrium, with the dissipative contribution $Y_{\alpha}$ vanishing, this equation simply states the fact that the initial coordinate $a_{\alpha}$ of a mass point does not change when one moves with it.
The entropy production $\dot{s}+\nabla_{i}\left(s v_{i}-f_{i}\right)=R / T$, conservation of mass and momentum, $\dot{\rho}+\nabla_{i}\left(\rho v_{i}\right)=0$, $\dot{g}_{i}+\nabla_{j}\left(\sigma_{i j}-\sigma_{i j}^{\mathrm{D}}\right)=0$, and Eq. (7) represent the complete hydrodynamic theory of solids, where

$$
\begin{array}{r}
\sigma_{i j}=p \delta_{i j}+v_{i} g_{j}+\psi_{\alpha j} \nabla_{i} a_{\alpha}, \\
R=f_{i} \nabla_{i} T+\sigma_{i j}^{\mathrm{D}} A_{i j}-Y_{\alpha} \nabla_{k} \psi_{\alpha k},
\end{array}
$$

[with $\left.A_{i k} \equiv \frac{1}{2}\left(\nabla_{i} v_{k}+\nabla_{k} v_{i}\right), p \equiv-\epsilon+T s+\mu \rho+v_{i} g_{i}\right]$ are unambiguously given by thermodynamics, Eq. (6), via the hydrodynamic standard procedure. Eq. (9) implies that the three fluxes $f_{i}, \sigma_{i j}^{\mathrm{D}}, Y_{\alpha}$ are linear combinations of the three forces $\nabla_{i} T, A_{i j}, \nabla_{k} \psi_{\alpha k}$. These give rise, respectively, to the dissipative phenomena of diffusive heat current, viscous stress, and defect diffusion. The structure of the linear combination, ie the symmetry of the Onsager coefficients, are given by the symmetry group of the crystal [8]. For isotropic solids, we have only diagonal terms, especially $f_{i} \sim \nabla_{i} T$ and $Y_{\alpha} \sim \nabla_{k} \psi_{\alpha k}$. As discussed at length in [1,9], it is incorrect to take the latter contribution as zero: The initial coordinate of a mass point may change, $\dot{a}_{\alpha} \neq 0$, in the absence of any mass current, $v_{i}=0$, when there is diffusive motion of vacancies. Conversely, motions of interstitials involve mass current, $v_{i} \neq 0$, but no change of crystal points, $\dot{a}_{\alpha}=0$.

Since $\nabla_{i} a_{\alpha}$ as a variable is completely equivalent to $U_{i j}$ and $R_{\alpha i}$, the equation of motion (17) for $\dot{a}_{\alpha}$ may always be rewritten as two equations of motion, for $\dot{U}_{i j}$ and $\dot{R}_{\alpha i}$. Though rather more complicated, this is certainly closer to the conventional elasticity theory. With the help of Eqs. (2) and (3), we rewrite Eq. (7) as

$$
\begin{array}{r}
2 \frac{\mathrm{d}}{\mathrm{dt}} U_{i j}=\left[\Xi_{j l} \Xi_{l k} \nabla_{i} v_{k}+R_{\alpha k} \Xi_{j k} \nabla_{i} Y_{\alpha}\right]+[i \leftrightarrow j], \\
\Xi_{i j} R_{\alpha l} \frac{\mathrm{d}}{\mathrm{dt}} R_{\alpha j}=-\Xi_{l j} \nabla_{i} v_{j}-R_{\alpha l} \nabla_{i} Y_{\alpha}-\frac{\mathrm{d}}{\mathrm{dt}} \Xi_{i l},
\end{array}
$$

which may be approximated by taking $U_{i j}, A_{i j}, Y_{\alpha}$ and $R_{\alpha j} \frac{\mathrm{d}}{\mathrm{d} t} R_{\alpha i}-\omega_{i j}$ as small quantities (with $2 \omega_{i j} \equiv \nabla_{j} v_{i}-$ $\left.\nabla_{i} v_{j}\right)$. To second order in the small quantities, though neglecting terms of order $U_{k j} \nabla_{i} Y_{\alpha}$, the result is

$$
\begin{gathered}
\frac{\mathrm{d}}{\mathrm{dt}} U_{i j}-A_{i j}=\left[\frac{1}{2}\left(\nabla_{i} Y_{\alpha}\right) R_{\alpha j}-\left(\nabla_{i} v_{k}\right) U_{k j}\right]+[i \leftrightarrow j] \\
R_{\alpha j} \frac{\mathrm{d}}{\mathrm{dt}} R_{\alpha i}-\omega_{i j}=\left[\frac{1}{2} R_{\alpha i} \nabla_{j} Y_{\alpha}+\frac{1}{2} U_{j k} A_{i k}\right]-[i \leftrightarrow j]
\end{gathered}
$$

Written in the conjugate variables of $U_{i j}$ and $R_{\alpha i}$, the stress tensor Eq. (8) reads

$$
\begin{aligned}
\sigma_{i j}= & p \delta_{i j}+v_{i} g_{j}-\Psi_{i j}+\Psi_{k i} U_{j k}+\Psi_{k j} U_{i k} \\
& +\chi_{\alpha j} R_{\alpha i}+\frac{1}{2} \chi_{\alpha k}\left(U_{k i} R_{\alpha j}+U_{k j} R_{\alpha i}\right) .
\end{aligned}
$$

This ends the presentation of the hydrodynamic theory of solids. The noteworthy point is: The derivation is completely cogent, as not a single step in it is discretionary; hence the above set of differential equations, given between $\mathrm{Eq}(6)$ and (9), is unique - any other theory is either algebraically equivalent, or wrong. Conversely, these equations account for any solid system, including crystals of all symmetry groups and glasses. This 
pertains especially to the nonlinear structure, important if one is to account for large displacement and rotation, strong compression and shear. These are usually small in bulk crystals, but quite large in complex liquids. In awareness of this, many nonlinear models for convectivelike nonlinearities have been suggested [6, 4], though none was constructed to contain the nonlinear solid limit.

To generalize our results to visco-elastic NonNewtonian fluids, we note first that the solid hydrodynamics contains the liquid hydrodynamics, and one can reduce the former to the latter by setting to zero the elastic stress $\psi_{\alpha i}=\partial \epsilon / \partial\left(\nabla_{i} a_{\alpha}\right)$. Confining ourselves to isotropic systems, it suffices to set $\Psi_{i j}=0$, because $\chi_{\alpha i}=0$ already holds. Taking $U_{i j}$ as the variable that relaxes as long as $\Psi_{i j} \neq 0$, we connect the isotropic solid dynamics to fluid dynamics such that the former holds in the high frequency regime (where the relaxation is negligible) and the latter in the low frequency regime (where relaxation is dominant). Therefore, we proceed by allowing a relaxation term $X_{i j}$ in Eq. (12), $\dot{U}_{i j}+\cdots=X_{i j}$. It leads to an additional term in the entropy production, Eq (9), $R=\cdots-X_{i j} \Psi_{i j}$, which implies $X=-\sum \alpha_{i} \Psi^{i}$ in an expansion, or to lowest order, $X_{i j}^{0}=-\alpha_{T} \Psi_{i j}^{0}$ and $X_{k k}=-\alpha_{L} \Psi_{l l}$. (The superscript ${ }^{0}$ denotes the traceless part of the given tensor.) So we have

$$
\begin{aligned}
\frac{\mathrm{d}}{\mathrm{d} t} U_{i j} & -A_{i j}+\left[\left(\nabla_{i} v_{k}\right) U_{k j}-\frac{1}{2}\left(\nabla_{i} Y_{\alpha}\right) R_{\alpha j}+i \leftrightarrow j\right] \\
& =-\alpha_{T} \Psi_{i j}^{0}-\alpha_{L} \Psi_{k k} \delta_{i j} / 3
\end{aligned}
$$

with $\alpha_{T}, \alpha_{L}$ denoting two transport coefficients. To understand the added terms, one can use the example of the harmonic approximation, Eq (屯), yielding $\alpha_{T} \Psi_{i j}^{0}=$ $\alpha_{T} K_{T} U_{i j}^{0}=U_{i j}^{0} / \tau_{T}$ and $\alpha_{L} \Psi_{k k}=\alpha_{L} K_{L} U_{k k}=U_{k k} / \tau_{L}$. Clearly, this implies relaxation for $U_{i j}^{0}$ and $U_{k k}$, with the respective relaxation times $\tau_{T}$ and $\tau_{L}$. (In principle, there are two thermodynamic cross derivatives, $\delta \Psi_{k k}=K_{\rho} \delta \rho+K_{T} \delta T$.)

Note the universality of the results, especially the convective terms $\sim\left(\nabla_{i} v_{k}\right)$, which remarkably are not preceded by any material-dependent coefficients. Their form is independent from the above approximation for $X_{i j}$ and will remain unchanged even if additional variables are introduced, eg to account for the material-dependent rheological behavior such as shear thinning and normal stress differences.

To account for large deformation, rotation and velocity, many different nonlinearities, as mentioned, have been suggested and implemented in the rheology literature, of which the two more popular ones are the upper and lower convective derivatives. Denoting an arbitrary matrix as $(*)$, the former is defined as $\hat{D}_{u}(*) \equiv(\partial / \partial t+\boldsymbol{v} \cdot \boldsymbol{\nabla})(*)+$ $(\boldsymbol{\nabla} \boldsymbol{v})(*)+(*)(\boldsymbol{\nabla} \boldsymbol{v})^{T}$, the latter as $\hat{D}_{\ell}(*) \equiv(\partial / \partial t+$ $\boldsymbol{v} \cdot \boldsymbol{\nabla})(*)-(\boldsymbol{\nabla} \boldsymbol{v})^{T}(*)-(*)(\boldsymbol{\nabla} \boldsymbol{v})$. Both are derived by invoking some variant of a postulated general principle, usually referred to as the "material frame independence". In the rheology literature [6,7], $(*)$ is the stress tensor, taken as independent, but in principle it could also be the strain tensor.
Reviewing the many equations of motion considered above, it is easy to see that Eqs. (12) and (15) for $U_{i j}$ can indeed be written as $\hat{D}_{u} \mathbf{U}-\mathbf{A}=O(\boldsymbol{\nabla} \boldsymbol{Y}, \boldsymbol{\Psi})$. None of the other equations may be brought into this form: It is not valid for $\dot{a}_{\alpha}$ and $\dot{R}_{\alpha i}$, see Eqs. (77, 11, 13); nor for the exact equations $\dot{U}_{i j}$, Eq. (10). Especially, it does not hold for the stress tensor.

This is a rather serious shortcoming and subjects all those descriptions that include upper and lower convected derivatives, combinations thereof, or other kinds of quadratic nonlinearities to grave doubts. To overcome these, the authors really need to convincingly argue why their postulated general principle overrules the simple and physical requirement that, for infinite yield time of the stress, the dynamics of non-Newtonian liquids such as polymer melts is that of an isotropic elastic medium.

[1] P.C. Martin, O. Parodi, and P.S. Pershan, Phys. Rev. A6, 2401 (1972).

[2] D. Forster, Hydrodynamic Fluctuations, Broken Symmetry and Correlation Functions (Benjamin, NY, 1975).

[3] L.D. Landau and E.M. Lifshitz, Hydrodynamics (Pergamon Press, Oxford, 1959).

[4] H. Pleiner and H.R. Brand in Pattern Formation in Liquid Crystals (A. Buka and L. Kramer, Eds., Springer, NY, 1996); T.C. Lubensky, Phys. Rev. A6, 452 (1972).

[5] R. Graham, Phys. Rev. Lett. 33, 1431 (1974); R. Graham and H. Pleiner, ibid. 34, 792 (1975); M. Liu, ibid. 43, 1740 (1979); H. Brand, M. Dörfle, and R. Graham, Ann. Phys. (N.Y.) 119, 434 (1979).

[6] C. Truesdell, Continuum Mechanics, Vols. 1 and 2 (Gordon and Breach, New York, 1962, 1965).

[7] R.B. Bird, R.C. Armstrong, and O. Hassager, Dynamics of Polymeric Liquids, Vol.1 (John Wiley \& Sons, New York, 1977); W. Noll, Arch. Rat. Mech. Anal. 2, 197 (1958); R.G. Larson, Constitutive Equations for Polymer Melts and Solutions (Butterworths, Boston, 1988); B.D. Coleman and W. Noll, Rev. Mod. Phys. 33, 239 (1961); J.G. Oldroyd, Proc. Roy. Soc. A200, 523 (1950); H. Giesekus, Rheol. Acta 5, 29 (1966).

[8] L.D. Landau and E.M. Lifshitz, Theory of Elasticity (Pergamon Press, Oxford, 1986).

[9] P.D. Fleming and C. Cohen, Phys. Rev. B13, 500 (1976).

[10] I.E. Dyaloshinskii and G.E. Volovik, Ann. Physics 125 67 (1980); B.J. Plohr and D.H. Sharp, Adv. Appl. Math. 9481 (1988); A.F. Andreev, Y.B. Bazaliy and A.D. Savishev, J. Low Temp. Phys. 88 12, (1992).

[11] P.M. Chaikin and T.C. Lubensky, Principles of condensed matter physics, Cambridge University Press, Cambridge, 1995, Chap. 6.5;

[12] H. Temmen, Die dissipative Dynamik elastischer und polarisierbarer Medien, PhD thesis Universität Hannover, (Shaker, Aachen, 1997). 